\title{
Extraction and quantification of levoglucosan and anhydrosugar isomers in sediments with ligand- exchange HPLC-ESI-MS/MS
}

\author{
DR. NINA DAVTIAN ${ }^{1}$, JOAN VILLANUEVA ${ }^{1}$, CARLES \\ MOREU $^{1}$, MARIA RAJA ${ }^{1}$ AND ANTONI ROSELL-MELÉ ${ }^{1,2}$ \\ ${ }^{1}$ Institut de Ciència i Tecnologia Ambientals (ICTA), Universitat \\ Autònoma de Barcelona (UAB) \\ ${ }^{2}$ Institució Catalana de Recerca i Estudis Avançats (ICREA) \\ Presenting Author: nina.davtian@uab.cat
}

Levoglucosan and its anhydrosugar isomers (galactosan and mannosan; MAS) are natural compounds generated from the pyrolysis of cellulose during biomass burning of continental vegetation. MAS are useful to identify and track emissions from modern and past wildfires, which are important to understand climate change and biogeochemical cycling processes. After atmospheric transport via biomass burning aerosols, MAS can be preserved in lake and marine sediments. However, MAS are not as frequently analyzed in sediments as they are in modern biomass burning aerosols. In addition, levoglucosan is typically the only anhydrosugar isomer analyzed in sediments given its higher abundance. For multi-biomarker analyses in sediments, published protocols typically only use a mixture of dichloromethane:methanol as the extraction solvent, which may be suboptimal for MAS given the high polarity of these compounds. Another shortcoming for analysis of all MAS is the scarcity of liquid chromatography methods that properly separate all isomers.

Here we describe a new method for MAS extraction and analysis in lake and marine sediments. Our new method employs ligand-exchange chromatography which achieves baseline separation for optimal MAS analysis. Our new method also has improved sensitivity, which helps us to detect and quantify anhydrosugar isomers much less abundant than levoglucosan in sediments. Using several organic solvents of contrasting polarities for biomarker extraction, we show that the polarity of the extraction solvent affects the recovery of MAS from sediments. For this reason, we recommend to systematically check whether the typically used 9:1 dichloromethane:methanol solvent mixture for biomarker extraction optimizes MAS recovery. In addition, we test several sample workout protocols to optimize MAS clean up before analysis in parallel with other compounds, such as benzene polycarboxylic acids derived from the chemical oxidation of pyrogenic carbon (BPCAs) and polyaromatic hydrocarbons (PAHs) as complementary biomass burning biomarkers. 